\title{
Ectopic Ascending Colonic Variceal Bleeding Treated with Balloon-Occluded Retrograde Transvenous Obliteration in a Decompensated Liver Cirrhosis Patient-A Hepatology Perspective
}

\author{
Gim Hin Ho ${ }^{*}$ (D), Suresh Babu², Chern Hao Chong3, Constantinos P. Anastassiades ${ }^{4}$ \\ ${ }^{1}$ Division of Gastroenterology, Department of Medicine, Khoo Teck Puat Hospital, Singapore \\ ${ }^{2}$ Department of Diagnostic Radiology, Interventional Radiology, Khoo Teck Puat Hospital, Singapore \\ ${ }^{3}$ Gut Care Digestive Liver Endoscopy Associates, Singapore \\ ${ }^{4}$ Division of Gastroenterology and Hepatology, Department of Medicine, National University Hospital, Singapore \\ Email: ${ }^{\star h o . g i m . h i n @ k t p h . c o m . s g ~}$
}

How to cite this paper: Ho, G.H., Babu, S., Chong, C.H. and Anastassiades, C.P. (2020) Ectopic Ascending Colonic Variceal Bleeding Treated with Balloon-Occluded Retrograde Transvenous Obliteration in a Decompensated Liver Cirrhosis Patient-A Hepatology Perspective. Case Reports in Clinical Medicine, 9, 191-200.

https://doi.org/10.4236/crcm.2020.97027

Received: June 6, 2020

Accepted: July 10, 2020

Published: July 13, 2020

Copyright $\odot 2020$ by author(s) and Scientific Research Publishing Inc. This work is licensed under the Creative Commons Attribution International License (CC BY 4.0).

http://creativecommons.org/licenses/by/4.0/

\section{(c) (i) Open Access}

\begin{abstract}
Ectopic colonic varices development from liver cirrhosis and portal hypertension is uncommon. They are part of the spectrum of portal hypertensive colopathy. Colonic variceal bleeding remains a rare cause of lower gastrointestinal tract (GI) bleeding. Due to the paucity of cases, there are no well-established conventional treatments for bleeding colonic varices. Different treatments have been reported. Here, we report a case of a 55-year-old gentleman, with a history of alcoholic liver cirrhosis, presenting with severe lower GI bleeding and symptomatic anaemia. An esophagogastroduodenoscopy revealed large esophageal varices with high-risk bleeding stigmata requiring endoscopic variceal ligation. A cross-sectional computed tomography scan showed colonic portosystemic shunts. In light of this and that the severe lower GI bleeding seemed out of proportion to the esophageal varices seen on upper endoscopy, an urgent unprepped colonoscopy was performed which revealed possible bleeding diverticula disease which required endoscopic mechanical hemoclip therapy. However, despite this, patient had recurrence of lower GI bleeding prompting a second colonoscopy. This relook colonoscopy showed ectopic ascending colon varices with high-risk bleeding stigmata. High-dose intravenous vasoactive agent somatostatin (500 mcg/hour) and subsequently terlipressin ( $2 \mathrm{mg}$ every 4 hours) were used. The patient subsequently underwent successful balloon-occluded retrograde transvenous
\end{abstract}


obliteration (B-RTO) and sclerotherapy. The non-selective beta-blocker (NSBB) carvedilol was started and bridged together with the vasoactive agent until stabilisation of portal hypertension. This difficult case illustrates the dynamic nature of portal hypertensive bleeding. It also highlights the presence of confounding non-variceal pathology complicating diagnosis of portal hypertensive colonic variceal bleeding, and that ectopic ascending colonic variceal bleeding can be treated successfully with B-RTO and sclerotherapy, with meticulous titration of high-dose vasoactive agents and NSBB, in a decompensated alcoholic liver cirrhosis patient.

\section{Keywords}

Ectopic Varices, Colonic Varices, Portal Hypertension, Balloon-Occluded Retrograde Transvenous Obliteration, Sclerotherapy, Liver Cirrhosis

\section{Introduction}

Varices formation is a complication of portal hypertension, with liver cirrhosis being the most common cause. Variceal bleeding is a common etiology of gastrointestinal (GI) bleeding in cirrhotic patients. Majority of varices are usually located in the esophagus and stomach. Ectopic varices, which are varices not in the esophagus or stomach, account for about 5\% of GI bleeding [1]. Amongst ectopic varices, colonic varices are rare, compared with duodenal and rectal varices, with a reported incidence of $0.07 \%$ [2]. They form part of the spectrum of portal hypertensive colopathy. Non-variceal causes like diverticula disease and haemorrhoids are common etiologies of lower GI bleeding. Ectopic colonic varices remain a rare cause of lower GI bleeding in cirrhosis patients. However, they do need to be suspected in cirrhosis patients who present with symptoms of lower GI bleeding. Unlike esophageal and gastric varices, there are no standard established therapeutic strategies for colonic varices [3]. This is largely due to the paucity of studies to evaluate ectopic colonic variceal bleeding due to the rare nature of the disease. Several therapies, including percutaneous transhepatic obliteration, colonic resection, portacaval shunt construction, transjugular intrahepatic portosystemic shunt (TIPSS), variceal embolization, and B-RTO, have been reported [4]. In this case, we successfully treated a case of ascending colonic variceal bleeding using B-RTO and sclerotherapy, with high-dose vasoactive agents and NSBB. B-RTO is an interventional radiological procedure usually performed via the renal vein, which allows the targeting and identification of the vessels responsible for feeding the varices [3]. This enables the inflation of the microballoon to occlude the blood flow of the varices to allow injection of sclerotherapy to facilitate thrombosis of the varices [3]. B-RTO is a local therapy which obliterates the culprit varices. However, portal hypertension remains the underlying mechanism of ectopic colonic varices formation. Higher portal pressures increase the risks of ectopic variceal formation and rupture. Intravenous 
vasoactive agents like somatostatin or terlipressin serve to lower portal pressures during an acute portal hypertensive variceal bleed. Oral NSBB like carvedilol or propranolol are subsequently employed to maintain low portal pressures as secondary prophylaxis to prevent subsequent bleeding episodes. Here, we report an interesting and difficult case of ectopic ascending colonic variceal bleeding in an alcoholic cirrhosis patient treated successfully using B-RTO and sclerotherapy, with meticulous titration of high-dose vasoactive agents and NSBB.

\section{Case Report}

Patient is a 55-year-old Chinese gentleman, who is an active chronic alcoholic. His co-morbidities include hyperlipidaemia, peripheral neuropathy from chronic alcoholism and alcoholic liver cirrhosis (Childs-Pugh-Turcotte score B7) with non-bleeding moderate-size esophageal varices and portal hypertension. He was previously on propranolol for primary prophylaxis. However, he has defaulted his follow-up appointments, surveillance and medications.

He presented to our hospital with a 2-day history of large volume hematemesis, associated with postural non-vertiginous giddiness and maroon coloured stools.

In the emergency department, his vital signs were; blood pressure 135/98 $\mathrm{mmHg}$, heart rate 101 beats per minute, temperature $36.7^{\circ} \mathrm{C}$. On examination, he was alert with a Glasgow coma scale of 15 and was jaundiced. He did not exhibit asterixis. He had clubbing but no other stigmata of chronic liver disease. Abdominal examination revealed hepatomegaly with no splenomegaly or ascites. A per-rectal examination showed maroon stains.

His laboratory results on admission are shown in Table 1.

Intravenous (IV) esomeprazole $8 \mathrm{mg}$ /hour infusion, IV somatostatin 250 mcg/hour infusion and IV ceftriaxone $1 \mathrm{~g}$ were initiated. An esophagogastroduodenoscopy (EGD) was performed, which revealed 4 columns of large esophageal varices with red wale sign and portal hypertensive gastropathy. Endoscopic variceal ligation was performed for the esophageal varices.

Table 1. Laboratory results on admission.

$\begin{array}{cccc}\begin{array}{c}\text { Hemoglobin } \\ \text { Platelets }\end{array} & 6 \text { g/dL (normal: } 13.1-17.4) & \text { Bilirubin } & 50 \text { umol/L }(3-21) \\ \text { WBC } & 134 \times 10^{9} / \mathrm{L}(173-414) & \text { Albumin } & 25 \mathrm{~g} / \mathrm{L}(35-50) \\ \text { Haematocrit } & 18 \%(34.4-45.3) & \text { GGT } & 121 \mathrm{U} / \mathrm{L}(11-50) \\ \text { PT } & 10.8 \text { seconds }(9.7-11.6) & \text { ALP } & 91 \mathrm{U} / \mathrm{L}(45-122) \\ \text { INR } & 1.03 & \text { Podium } & 137 \mathrm{mmol} / \mathrm{L}(135-145) \\ \text { APTT } & 25.1 \text { seconds }(25.5-36.8) & \text { Creatinine } & 5.7 \mathrm{mmol} / \mathrm{L}(3.5-5.1) \\ \text { ALT } & 59 \mathrm{U} / \mathrm{L}(10-44) & & \\ \text { AST } & 104 \mathrm{U} / \mathrm{L}(10-34) & & \end{array}$

PT-Prothrombin time, INR-International normalized ratio, APTT-Activated partial thromboplastin time, WBC-White blood cell; ALT-Alanine aminotransferase, AST-Aspartate aminotransferase, ALP_Alkaline phosphatase, GGT_Gamma glutamyl transpeptidase. 
However, immediately post EGD, patient developed large volume active hematochezia. As clinical symptoms seem inconsistent with the EGD findings, a lower GI bleeding pathology was considered. Patient underwent a computed tomography mesenteric angiogram (CTMA) which showed hyperdense contents in the colon and several right colonic and cecal diverticula without active arterial extravasation. There were also multiple portosystemic collaterals around the hepatic flexure and ascending colon (Figure 1). In addition, there was a partial segmental thrombosis of the main portal vein.

In light of these findings, a diagnostic dilemma of diverticular bleeding or a portal hypertensive colonic variceal bleed was considered. A colonoscopy was performed but the bowel preparation was poor. Intubation into the terminal ileum did not reveal any blood. Although there were no obvious active bleeding lesions, the blood appeared more pronounced in the cecum and ascending colon, correlating with the anatomic location of the right-sided diverticula. 2 suspicious diverticula with blood clots within were considered possible culprit lesions and decision was made to intervene with mechanical clip hemostasis (Figure 2). Within the limits of this index colonoscopy, no colonic or rectal varices were seen.

IV somatostatin was continued and transited to oral propranolol after 3 days. However, within hours of this transition, patient developed recurrent hematochezia with a significant drop in hemoglobin to $5.9 \mathrm{~g} / \mathrm{dL}$. A repeat CTMA showed

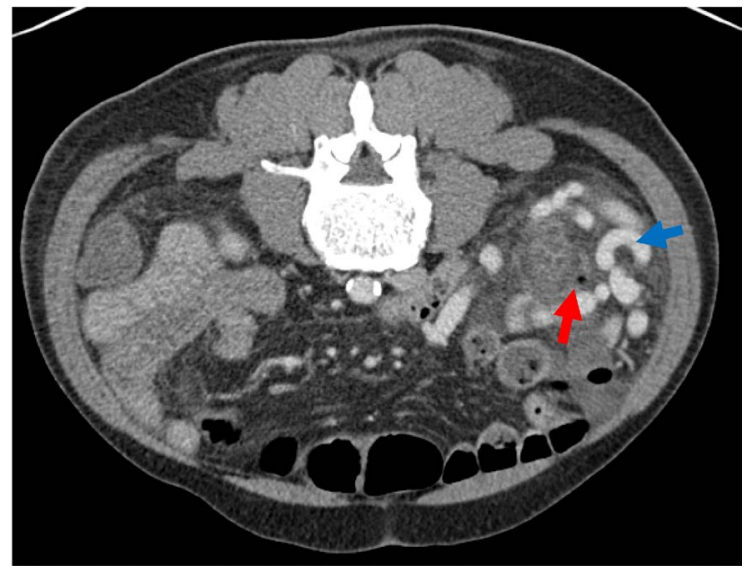

Figure 1. CTMA showing right colonic diverticula (red arrow) and portosystemic collaterals (blue arrow).
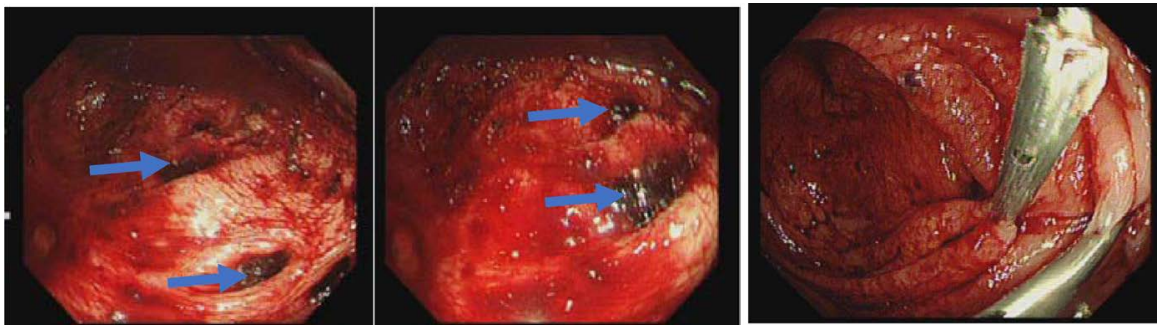

Figure 2. Multiple diverticula with blood clots within (no active bleeding) (blue arrows). Mechanical clips deployed over suspicious diverticula. 
stable multiple right colonic portosystemic collaterals with no active extravasation of blood (Figure 3).

With concerns of a colonic portal hypertensive variceal bleed, IV somatostatin was restarted at a higher dose of $500 \mathrm{mcg} / \mathrm{hour}$ and a second re-look colonoscopy was performed. The bowel preparation was significantly better. Deep terminal ileum intubation was negative for any active bleeding or stale blood. In the ascending colon, a large submucosa-appearing bulge was identified with possible white nipple sign (Figure 4(a)). It felt soft and fluctuant on probing with biopsy forceps. This was suggestive of a vascular lesion, consistent with the collaterals

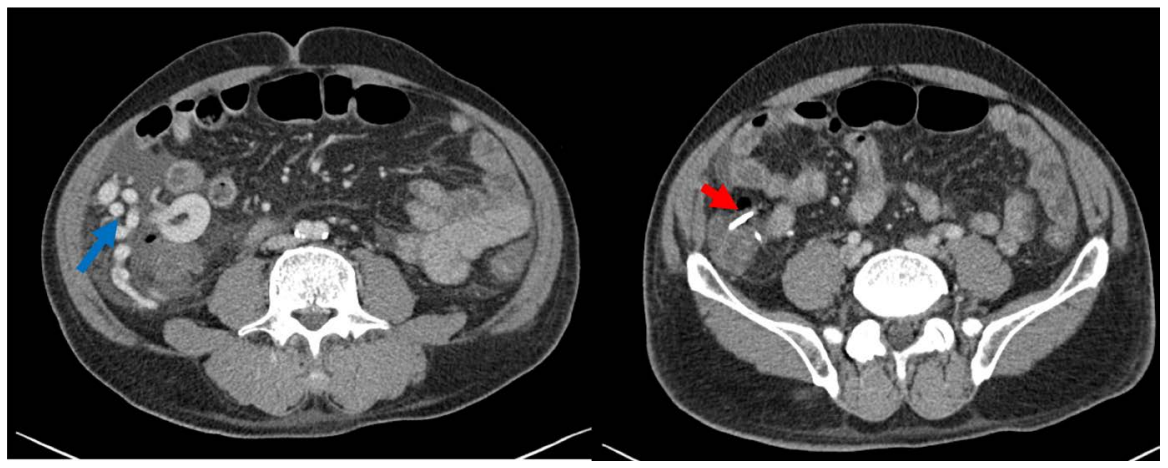

Figure 3. CTMA showing portosystemic collaterals (blue arrow) with hyperdense fluid in colon, without active bleeding. Previous right colonic diverticula clips seen (red arrow).

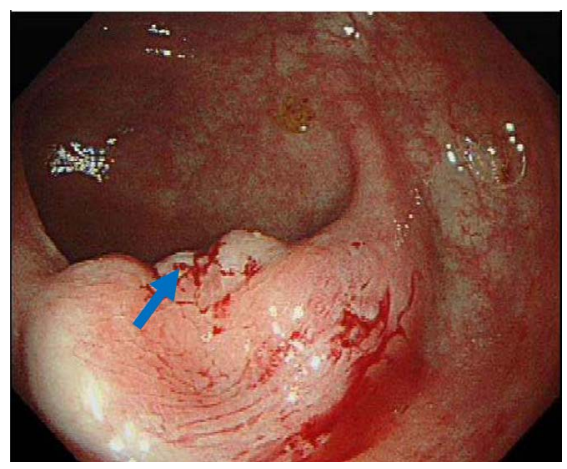

(a)
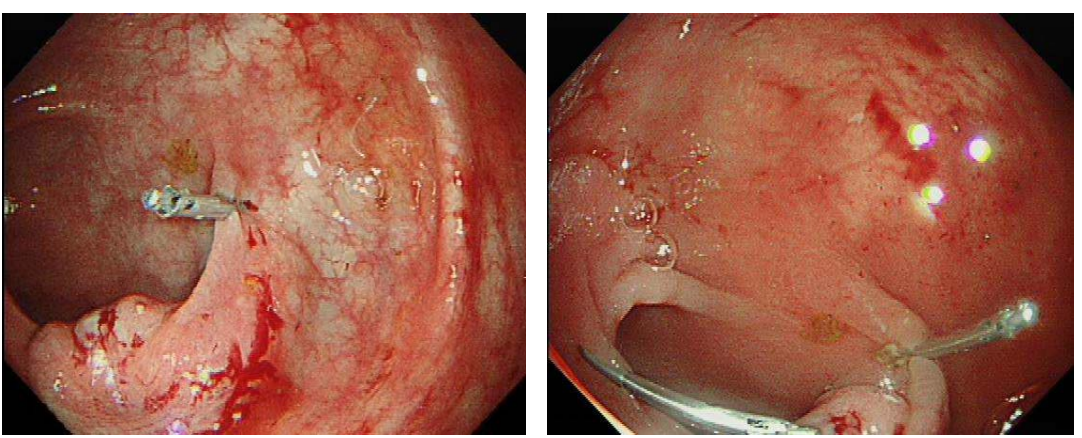

(b)

Figure 4. (a) Submucosal-appearing bulge suggestive of a vascular lesion with possible white nipple sign (blue arrow); (b) Mechanical hemoclips deployed at lateral ends of the vascular lesion for radiological localisation. 
seen on cross-sectional imaging. In the absence of active bleeding, no acute endoscopic intervention was performed. Instead, 2 mechanical hemoclips were deployed at the lateral ends of the vascular appearing lesion for subsequent interventional radiological localisation (Figure 4(b)).

High dose somatostatin was stopped and changed to terlipressin (2mg every 4 hours). A transthoracic echocardiogram revealed a preserved ejection fraction of $70 \%$, absence of right valvular heart disease and a normal pulmonary artery pressure of $34 \mathrm{mmHg}$. His model of end stage liver disease (MELD) score was 23 (driven predominately by acute renal impairment) and the Childs-Pugh-Turcotte score was A6.

After a multi-disciplinary discussion with interventional radiology and hepatobiliary surgeons, the consensus was to perform a B-RTO with sclerotherapy of the ectopic colonic varices, with consideration for TIPSS, in event B-RTO was unsuccessful.

Patient underwent the interventional radiological procedure via a right common femoral access. The wedged hepatic venous pressure (WHPG) was 24 $\mathrm{mmHg}$ and the free hepatic venous pressure was $9 \mathrm{mmHg}$. Hepatic venous pressure gradient (HVPG) was $15 \mathrm{mmHg}$. Taking into account patient had been on high-dose portal pressure lowering therapy. This was consistent with significant portal hypertension.

The images below show the balloon occlusion (Figure 5(a) and Figure 5(b), blue arrows) and injection of sclerotherapy with foamed sodium tetradecyl sulfate (Sotradecol, Mylan Pharma) and glue (Histacryl, B Braun) (Figure 5(a) and Figure 5(b), red arrows). Post-procedure, sclerosant is seen at the culprit varices (Figure 5(c), red arrow).

Terlipressin was stopped after 3 days because of development of asymptomatic hyponatremia. Propranolol was started. However, within 1 day of stopping terlipressin, hematochezia recurred.

A CTMA revealed large varices around the hepatic flexure and ascending colon with interval embolisation and resultant thrombosis in the dilated varices

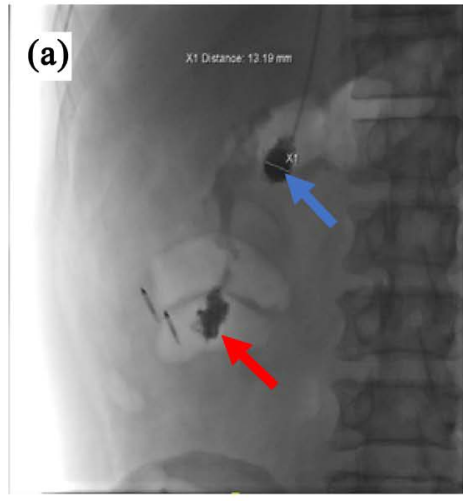

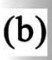

(b)

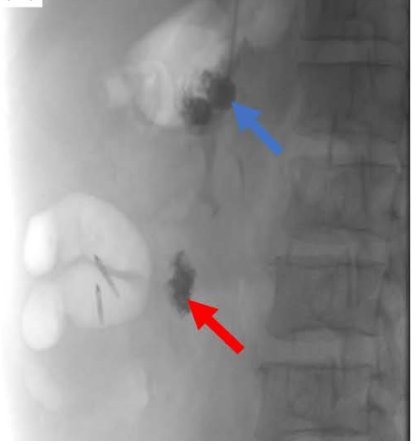

(c)

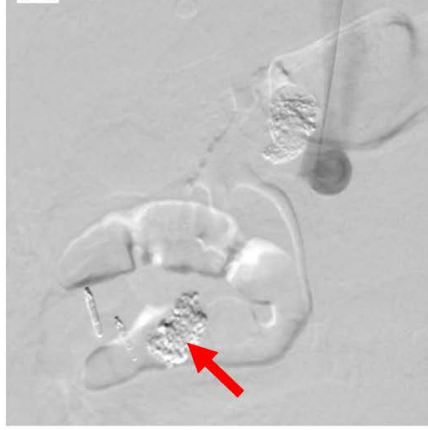

Figure 5. (a) and (b) Fluoroscopic views of balloon occlusion performed (blue arrow), contrast injected revealing varices with sclerotherapy performed (red arrow). (c) Post procedure image with sclerotherapy performed to culprit varices (red arrow). 
(Figure 6). High-dose (500 mcg/hour) somatostatin was restarted, with resolution of bleeding.

We hypothesized that this re-bleeding was possibly flash bleeding related to a transient rise in portal pressure when the intravenous vasoactive agent was transited to NSBB. In light of this, we bridged patient with carvedilol concurrently with high-dose somatostatin. Somatostatin was eventually stopped and carvedilol dose was gradually escalated to $18.75 \mathrm{mg}$ twice daily.

A surveillance CT done 6 weeks later showed thrombosis of the embolised colonic varix (Figure 7).

A surveillance colonoscopy 3 months later revealed resolution of the ectopic ascending colonic varix (Figure 8). A year on from his presentation, patient has quit alcohol consumption and is maintained on high-dose carvedilol. He remains asymptomatic with no reported GI bleeding, and his liver function test is normal.

\section{Discussion}

Ectopic colonic variceal bleeding remains an atypical cause of lower GI bleeding

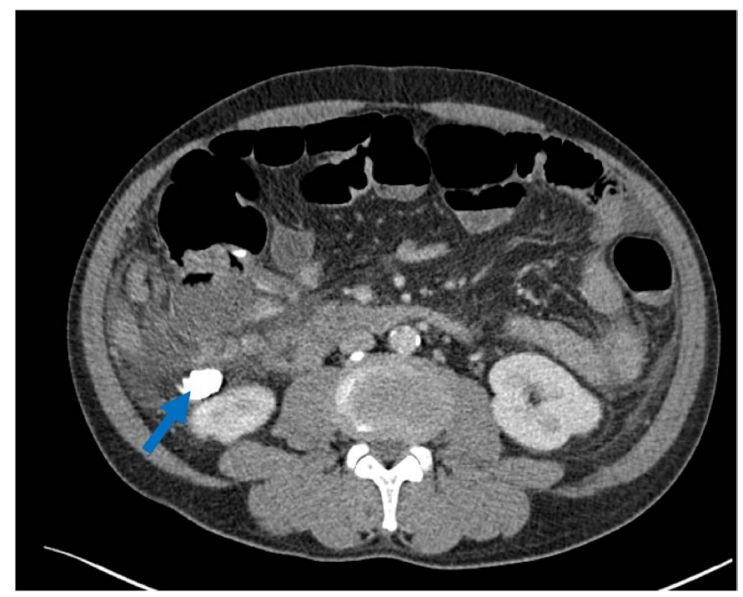

Figure 6. Varices with sclerosant thrombosis (blue arrow).

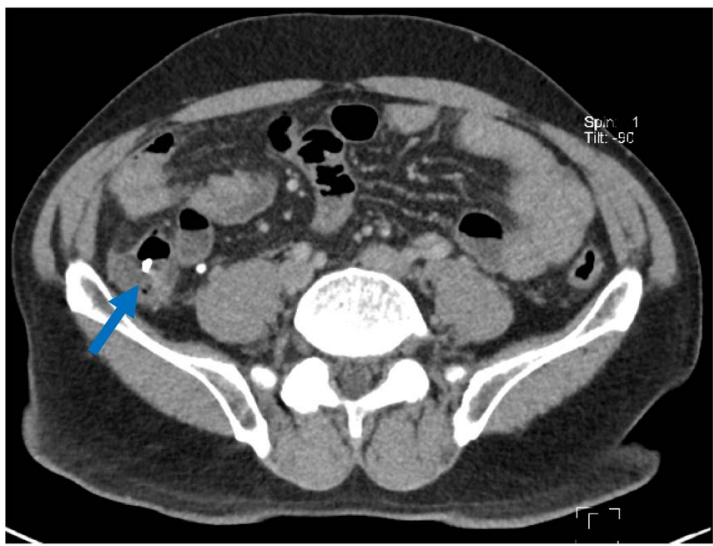

Figure 7. Thrombosed colonic varices 6 weeks post B-RTO and sclerotherapy (blue arrow). 


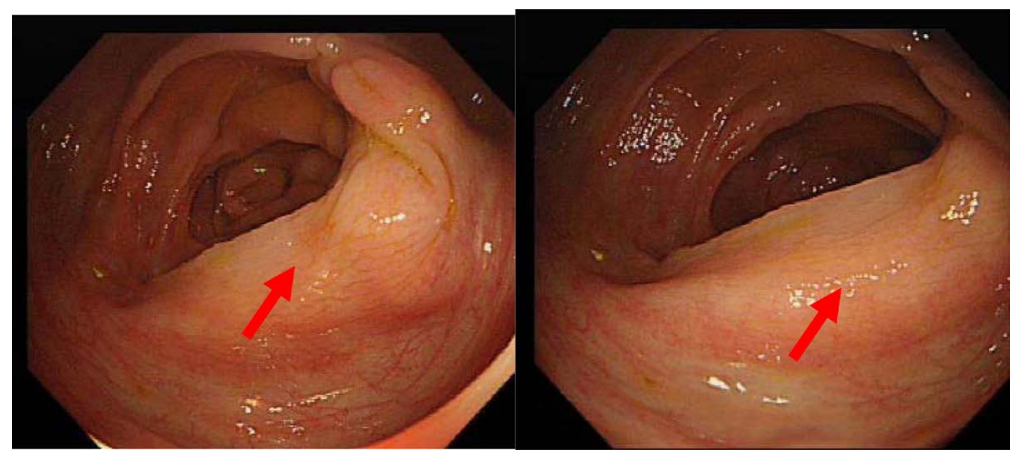

Figure 8. Resolution of the ectopic colonic varix (in red arrows) at surveillance colonoscopy 3 months post B-RTO and sclerotherapy.

in liver cirrhosis patients [5]. Despite this, they should still be considered in cases of lower GI bleeding in liver cirrhosis. Management of bleeding ectopic colonic varices is not well established due to the paucity of cases and studies regarding their treatment. Various strategies have been employed. These include percutaneous transhepatic obliteration, colonic resection, portacaval shunt construction and TIPSS [4]. However, these procedures have risks of liver decompensation. Therefore, alternative strategies are required.

B-RTO has been reported to provide good variceal bleeding control and rebleeding free survival [6]. However, these findings were obtained from studies on B-RTO in gastric variceal bleeding [6]. B-RTO is usually employed in patients with gastric variceal bleeding [3]. Similar to our case, Matsumoto et al. also employed B-RTO to successfully treat an ascending colon variceal bleed [3].

Treatment strategy for colonic varices is determined by various factors. These include underlying etiology of cirrhosis and portal hypertension, severity of the bleeding, distribution of the varices [7], size and accessibility of the portal vein and severity of liver disease.

In this case, we opted for B-RTO instead of TIPSS chiefly for 2 reasons. Firstly, the portal vein diameter was attenuated, making TIPSS technically more challenging. Secondly was the possibility of a TIPSS stent making liver transplant surgery more difficult. It has been reported that complications related to TIPSS stent can result in a technically more complex liver transplant surgery [8]. We considered the possibility of liver transplant in event of refractory bleeding to both interventional radiological and endoscopic therapies. In addition, performing B-RTO would not exclude the patient for TIPSS in the event of recurrent bleeding after B-RTO.

Despite the success of B-RTO in this case, it is important to note that B-RTO is a localised therapy which serves to obliterate the culprit varices. However, it does not reduce portal pressures like TIPSS or portal pressure lowering agents. Patients will require vasoactive agents and NSBB to target the underlying pathophysiology of the varices, which is portal hypertension. For this case, both terlipressin and high-dose somatostatin were used. However, terlipressin use was limited by the development of hyponatremia. Although several studies [9] [10] 
[11] showed no significant difference in rebleeding or mortality between terlipressin and somatostatin for variceal bleeding, Sarin et al. recommended the use of terlipressin in ectopic variceal bleed [12]. Villanueva et al. also reported that in non-responders to usual somatostatin dose, both terlipressin and high-dose somatostatin (500 mcg/hour) significantly decreased HVPG and increased the rate of hemodynamic responders [13]. These effects were greater with terlipressin compared to somatostatin [13]. In addition, this case also suggests that bridging vasoactive agent with NSBB concurrently for high risk and recurrent ectopic variceal bleeding may be a useful strategy to employ, although this needs further verification in a prospective study. It is important to re-iterate that TIPSS or liver transplant remains the treatment for refractory ectopic variceal bleeding.

\section{Conclusion}

In conclusion, this difficult case illustrates the dynamic nature of portal hypertensive bleeding. It also highlights the presence of confounding non-variceal pathology complicating diagnosis of portal hypertensive colonic variceal bleeding. Ectopic colonic variceal bleeding should be considered in lower GI bleeding in cirrhosis patients. B-RTO and sclerotherapy, with close titration of high-dose vasoactive agents and NSBB is an effective and safe option for the treatment of ectopic colonic variceal bleeding.

\section{Declaration}

Verbal and written informed consents were obtained from the patient.

\section{Conflicts of Interest}

The authors declare no conflicts of interest regarding the publication of this paper.

\section{References}

[1] Norton, I.D., Andrews, J.C. and Kamath, P.S. (1998) Management of Ectopic Varices. Hepatology, 28, 1154-1158. https://doi.org/10.1002/hep.510280434

[2] Han, J.H., Jeon, W.J., Chae, H.B., Park, S.M., Youn, S.J., Kim, S.H., Bae, I.H. and Lee, S.J. (2006) A Case of Idiopathic Colonic Varices: A Rare Cause of Hematochezia Misconceived as Tumour. World Journal of Gastroenterology, 12, 2629-2632. https://doi.org/10.3748/wjg.v12.i16.2629

[3] Matsumoto, K., Imai, Y., Takano, M., Nakazawa, M., Ando, S., Sugawara, K., Nakayama, N., Tomiya, T. and Mochida, S. (2018) A Case of Colonic Varices Complicated by Alcoholic Cirrhosis Treated Using Balloon-Occluded Retrograde Transvenous Obliteration. Clinical Journal of Gastroenterology, 11, 343-347. https://doi.org/10.1007/s12328-018-0857-1

[4] Sato, T., Akaike, J., Toyota, J., Karino, Y. and Ohmura, T. (2011) Clinicopathological Features and Treatment of Ectopic Varices with Portal Hypertension. International Journal of Hepatology, 2011, Article ID: 960720. https://doi.org/10.4061/2011/960720

[5] Sunkara, T., Caughey, M.E. and Culliford, G.V. (2018) Idiopathic Isolated Colonic 
Varices: An Extremely Rare Condition. Journal Clinical Medical Research, 10, 63-65. https://doi.org/10.14740/jocmr3230w

[6] Gimm, G., Chang, Y., Kim, H.C., et al. (2018) Balloon-Occluded Retrograde Transvenous Obliteration versus Transjugular Intrahepatic Portosystemic Shunt for the Management of Gastric Variceal Bleeding. Gut and Liver, 12, 704-713. https://doi.org/10.5009/gnl17515

[7] Van Geenen, E.M. and Geert, J.A. (2015) Right Sided Colonic Varices: 2 Cases and Review of the Literature. Journal of Gastrointestinal and Digestive System, 13, 009. https://doi.org/10.4172/2161-069X.S13-009

[8] Mazziotti, A., Morelli, M.C., Grazi, G.L., Jovine, E., Masetti, M., Pierangeli, F. and Cavallari, A. (1996) Beware of TIPS in Liver Transplant Candidates. Transjugular Intrahepatic Portosystemic Shunt. Hepatogastroenterology, 43, 1606-1610.

[9] Wells, M., Chande, N., Adams, P., Beaton, M., Levstik, M., Boyce, E. and Mrkobrada, M. (2012) Meta-Analysis: Vasoactive Medications for the Management of Acute Variceal Bleeds. Alimentary Pharmacology and Therapeutics, 35, 1267-1278. https://doi.org/10.1111/j.1365-2036.2012.05088.x

[10] Seo, Y.S., Park, S.Y., Kim, M.Y., Kim, J.H., Park, J.Y., Yim, H.J., Jang, B.K., Kim, H.S., Hahn, T., Kim, B.I., Heo, J., An, H., Tak, W.Y., Baik, S.K., Han, K.H., Hwang, J.S., Park, S.H., Cho, M. and Um, S.H. (2014) Lack of Difference among Terlipressin, Somatostatin and Octreotide in the Control of Acute Gastroesophageal Variceal Hemorrhage. Hepatology, 60, 954-963. https://doi.org/10.1002/hep.27006

[11] De Franchis, R. (2004) Somatostatin, Somtostain Analogues and Other Vasoactive Drugs in Treatment of Bleeding Esophageal Varices. Digestive and Liver Disease, 26, S93-S100. https://doi.org/10.1016/j.dld.2003.11.017

[12] Sarin, S.K. and Kumar, C.K.N. (2012) Ectopic Varices. Clinical Liver Disease, 1, 167-172. https://doi.org/10.1002/cld.95

[13] Villanueva, C., Planella, M., Aracil, C., Lopez-Balaguer, J.M., Gonzalez, B., Minana, J. and Balanzo, J. (2005) Hemodynamic Effects of Terlipressin and High Somatostatin Dose during Acute Variceal Bleeding in Nonresponders to the Usual Somatostatin Dose. American Journal of Gastroenterology, 100, 624-630. https://doi.org/10.1111/j.1572-0241.2004.40665.x 